\title{
Staging of the mediastinum: value of positron emission tomogra- phy imaging in non-small cell lung cancer
}

\author{
Th. Bury*, P. Paulus**, A. Dowlati*, J.L. Corhay*, T. Weber*, B. Ghaye ${ }^{+}$, \\ J. Schoffers ${ }^{+\#}$, R. Limet ${ }^{++}$, A. Albert $\$$ P. Rigo**, M. Radermecker*
}

Staging of the mediastinum: value of positron emission tomography imaging in nonsmall cell lung cancer. Th. Bury, P. Paulus, A. Dowlati, J.L. Corhay, T. Weber, B. Ghaye, J. Schoffers, R. Limet, A. Albert, P. Rigo, M. Radermecker. (ERS Journals Ltd 1996.

ABSTRACT: Recent studies have shown limitations of morphological imaging in staging mediastinal lymph node involvement in lung cancer. In contrast to computed tomography (CT), which depends primarily on anatomical imaging features, positron emission tomography (PET) with 18-fluorodeoxyglucose (FDG) depends mainly on the metabolic characteristics of a tissue for the diagnosis of disease.

We have performed a prospective study comparing FDG-PET and CT of the thorax in the presurgical assessment of the mediastinum in 50 patients with newly diagnosed non-small cell lung cancer (NSCLC). CT and PET scans were interpreted separately, and results were compared to pathological staging obtained during thoracotomy.

Hilar or mediastinal lymph node involvement was present in $58 \%$. In staging for lymph node involvement, CT had a sensitivity of $72 \%$ and specificity of $81 \%$, whereas PET had a sensitivity and specificity of $90 \%$ and $86 \%$, respectively. When the PET study was compared to histological results, there were four cases showing more advanced mediastinal involvement with PET and four cases showing less involvement with PET.

From our preliminary results, we conclude that positron emission tomography with 18-fluorodeoxyglucose is significantly more accurate than computed tomography in the mediastinal staging of non-small cell lung cancer.

Eur Respir J., 1996, 9, 2560-2564.

The most effective treatment of non-small cell lung cancer (NSCLC) is surgical resection. Surgical resectability depends, in large part, upon the presence and degree of mediastinal involvement [1, 2]. At present, computed tomography (CT) is the most effective noninvasive technique for the evaluation of mediastinal nodes [3]. However, its sensitivity and specificity are highly variable between studies depending on patient population, technology and diagnostic criteria [4-6].

We have carried out positron emission tomography (PET) in our centre since May 1994, using 18-fluorodeoxyglucose (18-FDG) as a routine radiopharmaceutical tracer to evaluate the increased glucose metabolism in tumour cells $[7,8]$. Indeed, a high rate of glycolysis is a biochemical hallmark of malignant tumours, and it is associated with increased activity of rate-controlling enzymes for glycolysis, including hexokinase, phosphofructokinase and pyruvate dehydrogenase [9, 10]. Preliminary studies have shown that FDG-PET can accurately differentiate benign from malignant pulmonary abnormalities $[10,11]$. In particular, it has a high degree of accuracy in differentiating between benign and malignant solitary pulmonary nodules [12-14]. We therefore performed a prospective study in 50 patients, to determine the value of 18-FDG-PET in the preoperative mediastinal assessment of non-small cell lung cancer.
Depts of *Pneumology, **Nuclear Medicine,

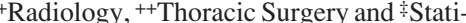
stics, CHU Liège, Belgium. ${ }^{\#} \mathrm{CHR}$, Verviers, Belgium.

\author{
Correspondence: Th. Bury \\ Pneumology Dept \\ CHU Sart Tilman, B35 \\ 4000 Liege \\ Belgium
}

Keywords: Computed tomography scan mediastinum staging

non-small cell lung cancer

positron emission tomography scan

Received: March 291996

Accepted after revision August 161996
Subjects and methods

\section{Subjects}

All patients with potentially resectable non-small cell lung cancer (NSCLC) who were examined in the pneumology department of our institution were considered for this investigation. The diagnosis of NSCLC was made by cytological study of sputum, needle biopsy of a lung nodule or hilar mass, or flexible bronchoscopy. Patients were excluded from the study if they had one of the following contraindications to surgery: inoperable tumours (T4 lesion); poor physiological condition (inadequate cardiopulmonary function); or metastases noted on preoperative evaluation (including isotope studies and abdominal and cerebral CT).

Up to the present time, 50 patients (mean age $65 \mathrm{yrs}$; range 44-75 yrs), with a new diagnosis of NSCLC, have been enrolled in this prospective study. The patients underwent CT scanning and PET imaging of the thorax during the 10 days before surgery (mediastinoscopy or thoracotomy). Histological diagnoses of pulmonary lesions were as follows: squamous cell carcinoma $(n=21)$; adenocarcinoma $(n=24)$; and undifferentiated large-cell carcinoma $(n=5)$ : The tumour was considered central when it was seen directly during fibreoptic bronchoscopy. Otherwise, it was considered peripheral. 


\section{CT scanning}

CT scanning was performed using a PQ2000 (fourth generation, Picker, Cleveland, OH, USA) instrument. Spiral volume was obtained from the lung apices to the posterior costophrenic angles, with a slice thickness of $10 \mathrm{~mm}$ and $8 \mathrm{~mm}$ reconstruction increment. In some cases, a spiral acquisition of $5 \mathrm{~mm}$ thick slice and $3 \mathrm{~mm}$ reconstruction increment were obtained in the region of the hili. A bolus of $120 \mathrm{~mL}$ iodinated contrast material (Optiray 300; Codali SA, Laboratoire Guerbert, Aulnay-Sous-Bois, France) was administrated in all cases.

CT images were read independently by two groups of two radiologists, who had knowledge of the standard chest radiographs and of the histological diagnosis of the primary tumour. Mediastinal lymph nodes were localized according to the lymph node mapping scheme of the American Thoracic Society (ATS) [15]. To classify lymph nodes, the short axis on the transaxial views was measured; if it exceeded $10 \mathrm{~mm}$, the node was considered diseased. At the end of this first stage of interpretation, the two teams of reviewers compared findings. In this study, there was no disagreement concerning CT scans.

\section{FDG-PET imaging}

Prior to the PET study, all patients fasted for $6 \mathrm{~h}$. PET was performed using a UGM Penn PET $240 \mathrm{H}$ scanner. This machine consists of six large areas $(50 \times$ $20 \mathrm{~cm}$ ) of NaI crystals of $2.5 \mathrm{~cm}$ thickness, in a hexagonal arrangement. The system has no septa or shadow shield but operates in 3D with an acceptance angle of 6.5 degrees. Opposed crystals are separated by $80 \mathrm{~cm}$, but the effective field of view is $512 \mathrm{~mm}$ in the $X$ and $\mathrm{Y}$ directions and $128 \mathrm{~mm}$ in the $\mathrm{Z}$ direction. Images are reconstructed as 32 transverse planes composed of $4 \mathrm{~mm}$ isotropic voxels to form a $128 \times 128$ matrix. It is also possible to reconstruct 64 transverse planes using $2 \mathrm{~mm}$ voxels but with a field of view limited to 256 $\mathrm{mm}$. Images are reconstructed using a Hanning filter. The effective spatial resolution of clinical data is $\approx 8$ $\mathrm{mm}$ (full width half maximum (FWHM)), while the intrinsic resolution of the machine using phantoms and a ramp filter is $5.5 \mathrm{~mm}$ (specification). Six to 10 steps $(512-768 \mathrm{~mm})$ extending from the neck to the abdomen, total scanning time $40-60 \mathrm{~min}\left(4-8 \mathrm{~min} \cdot \mathrm{step}^{-1}\right)$, are acquired for each patient to cover the thoracic area which is necessary for the pulmonary study. PET data were analysed by visual interpretation of coronal, sagittal and transverse slices alone and by cross-referencing.

PET images were read independently by two groups of two nuclear physicians, who had knowledge of the standard chest radiographs and of the histological diagnosis of the primary tumour. They evaluated the presence or the absence of 18-FDG uptake in the hilar or mediastinal area. They made no attempt to localize visualized activity in a particular nodal station, except for differentiating right from left and hilar from mediastinal activity. When increased uptake of 18-FDG was observed, two levels were identified: moderate or intense. At the end of the first step of interpretation, the two teams of reviewers compared their findings. When they did not agree, the teams reviewed the images together to reach a consensus. In this study, there were two instances of disagreement concerning PET images.

\section{Comparison of the results}

PET and CT imaging of the thorax were interpreted separately and, subsequently, results were compared with each other and with the surgical proof (mediastinoscopy, thoracotomy). Mediastinoscopy $(n=15)$ was performed in the majority of cases where "N2" or "N3" stage was suspected by CT or PET study. At thoracotomy $(n=46)$, all accessible nodes were sampled and examined on frozen section. Only nodes that underwent biopsy or resection were considered for analysis.

\section{Statistical analysis}

Diagnostic specificity and sensitivity of CT and PET imaging, in pooling results of surgery into two categories: absence (N0) or presence $(\mathrm{N} 1, \mathrm{~N} 2, \mathrm{~N} 3)$ of node involvement, have been calculated by the classical method. Positive and negative predictive values were also evaluated in the same manner. For each of these parameters, the $95 \%$ confidence interval $(95 \% \mathrm{CI})$ is given.

To assess the agreement between CT or PET and surgery, more precisely the value "Kappa" $(\kappa)$ of Cohen with its $95 \%$ CI was calculated on the same patients [16]. This $\kappa$ value allows comparison of the degree of agreement between the two methods. By this statistical method, the degree of agreement between two methods is better if the " $\kappa$ " value approaches the number 1 . To compare the diagnostic efficacy of CT and PET, we compared their $\kappa$ values by Chi-squared analysis. A pvalue of less than 0.05 was considered significant.

\section{Results}

\section{Clinical data}

Table 1 shows the final staging for the 50 patients according to tumour histology. The prevalence of hilar or mediastinal lymph node involvement was 58\% (29 of the 50 cases). There were 21 cases classified as N0, 13 as N1, 12 as N2 and 4 as N3. Of these lung cancers, there were 23 central tumours and 27 considered as peripheral tumours.

\section{Evaluation of nodal involvement}

Among the 50 cases, preoperative radiological staging proposed by CT scan and PET-FDG was similar in 29 cases: this evaluation was correct in 27 cases after obtaining histological proof (15 cases N0, 5 N1, 4 N2 and 3 N3) and overestimated in two cases. Table 2 summarizes the 21 discordant $\mathrm{CT}$ and PET results. The preoperative radiological (CT scan versus PET-FDG) staging was conflicting in 21 of the 50 cases: by comparison to histology, this clinical staging was correct in 15 cases by PET-FDG and in six cases by CT. 
Table 1. - Patient distribution according to "N" description and tumour histology

\begin{tabular}{lcccc}
\hline & $\mathrm{N} 0$ & $\mathrm{~N} 1$ & $\mathrm{~N} 2$ & $\mathrm{~N} 3$ \\
\hline $\begin{array}{l}\text { Squamous cell } \\
\text { carcinoma (n=21) }\end{array}$ & 12 & 4 & 4 & 1 \\
$\begin{array}{l}\text { Adenocarcinoma } \\
(\mathrm{n}=24)\end{array}$ & 9 & 6 & 6 & 3 \\
$\begin{array}{l}\text { Large cell carcinoma } \\
(\mathrm{n}=5)\end{array}$ & 0 & 3 & 2 & 0 \\
\hline Total $(\mathrm{n}=50)$ & 21 & 13 & 12 & 4 \\
\hline
\end{tabular}

"N": node classification.

By PET study, lymph node involvement was correctly identified in 24 out of 29 cases (10 N1 stage, 10 N2 stage, $4 \mathrm{~N} 3$ stage) and the absence of lymph node involvement in 18 out of 21 cases. By PET technique, four cases had a higher degree of mediastinal involvement compared to histology: one with histological evidence of lymph node involvement but to a lesser degree than that proposed by PET; and three with no histological lymph node involvement, but with proposed disease infiltration by PET study. On fibreoptic bronchoscopy, the tumour was central in these four cases and CT also suggested a lymph node involvement in two of the four. The mediastinal 18-FDG uptake was moderate in three of the cases, and as intense as the primary tumour in one case. In this last case, pathological examination showed enlarged anthracotic lymph nodes at the site of FDG uptake.

Table 2. - Comparative performance of computed tomography of (CT) and positron emission tomography (PET) in mediastinal staging in conflicting cases (21 out of 30 patients)

\begin{tabular}{rrrc}
\hline Pt & CT & PET & $\begin{array}{c}\text { Histological } \\
\text { finding }\end{array}$ \\
\hline 1 & N3 & N0 & N0 \\
2 & N1 & N0 & N0 \\
3 & N0 & N2 & N2 \\
4 & N0 & N1 & N1 \\
5 & N2 & N1 & N2 \\
6 & N0 & N1 & N1 \\
7 & N0 & N1 & N1 \\
8 & N2 & N0 & N2 \\
9 & N0 & N2 & N2 \\
10 & N3 & N0 & N0 \\
11 & N0 & N1 & N1 \\
12 & N0 & N3 & N3 \\
13 & N2 & N0 & N1 \\
14 & N1 & N2 & N2 \\
15 & N3 & N0 & N1 \\
16 & N1 & N2 & N2 \\
17 & N3 & N1 & N1 \\
18 & N2 & N2 & N2 \\
19 & N0 & N1 & N0 \\
20 & N0 & N2 & N2 \\
21 & N0 & & \\
\hline
\end{tabular}

This table shows all cases incorrectly classified by CT or PET, with the exception of two cases which were overestimated by both techniques. CT: computed tomography; PET: positron emission tomography; $\mathrm{N}$ : node classification.
In the PET-FDG series, four cases had a lesser degree of mediastinal involvement compared to histology: three with negative PET in spite of positive histology; and one with a positive PET but to a lesser degree than the histological involvement (table 2). In two of these four cases, the tumour was judged to be central and contiguous to the mediastinum; it was not possible to separate the 18-FDG uptake in the tumour from its extension in lymph nodes. The other two cases were peripheral tumours with either N1 or N2 disease. Figure 1 shows $\mathrm{CT}$ and PET-FDG images in a patient with peripheral adenocarcinoma. This patient was classified N0 by CT and N1 by PET-FDG. Nodal N1 metastasis was confirmed surgically.

The CT study correctly classified 16 out of 29 lymph node involvements ( $7 \mathrm{~N} 1$ stage, $6 \mathrm{~N} 2$ stage and $3 \mathrm{~N} 3$ stage), and 17 out of 21 N0 stage. Among the incorrect stages proposed by CT, eight cases had a higher degree of mediastinal involvement compared to histology, and

a)

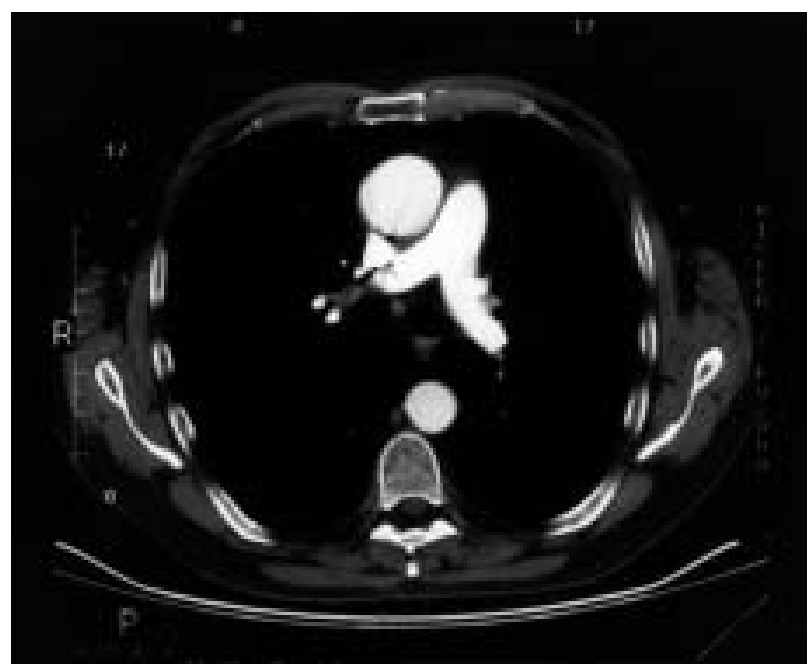

b)

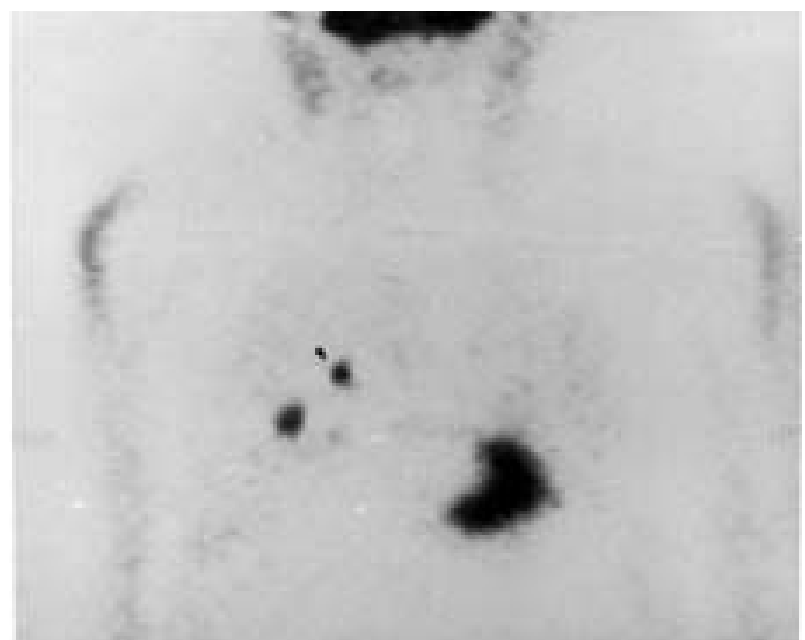

Fig. 1. - CT and PET-FDG images in a patient with peripheral adenocarcinoma. a) Absence of suspect lymph nodes on diagnostic contrast-enhanced CT scan. b) Coronal image showing the FDG uptake in the primary tumour (right lower lobe) and in the ipsilateral hilar area (arrow). Note also the FDG uptake by the heart (head of patient is at the top of the figure). CT: computed tomography; PETFDG: positron emission tomography with 18-fluorodeoxyglucose. 
nine had a lesser degree of mediastinal involvement compared to histology (table 2).

In mediastinal staging, PET-FDG had a sensitivity of $90 \%$ (95\% CI: $73-98 \%)$ and a specificity of $86 \%$ (95\% CI 64-97\%). The corresponding positive and negative predictive values were $90(95 \%$ CI 73-98\%) and $86 \%$ (95\% CI 64-97\%) respectively. By contrast, chest CT had a sensitivity of $72 \%$ (95\% CI 53-87\%), specificity of $81 \%$ (95\% CI 58-95\%), positive predictive value of $84 \%$ (95\% CI 64-95\%) and negative predictive value of $68 \%(95 \%$ CI $47-85 \%)$.

By comparing the " $\kappa$ " values of CT $(\kappa=0.50 ; 95 \%$ CI $0.34-0.67)$ and of PET ( $\kappa=0.77 ; 95 \%$ CI $0.59-0.94)$, we can conclude that the agreement between PET and surgical staging was significantly better than the agreement between CT and surgery $(\mathrm{p}=0.033)$.

\section{Discussion}

This study shows that PET-FDG imaging could be useful in assessing mediastinal disease in patients with a recent diagnosis of NSCLC. Sensitivity and specificity of the method were higher than CT in this indication, and hopefully technical advances in noninvasive mediastinal PET imaging will narrow the gap between clinical and surgical staging. In NSCLC, the preoperative evaluation of the mediastinum for lymph node metastasis is very important for correct staging and in predicting surgical resectability. At present, CT scan is a valuable method for intrathoracic staging of lung cancer, although the reported sensitivity of CT has varied in different studies [4-6, 17, 18]. Indeed, the diagnostic efficacy of CT shows large variation from one study to another, depending on patient selection, technology and diagnostic criteria. In general, the smaller the criterion for nodal diameter used to separate malignant from benign nodes, the higher the sensitivity and the lower the specificity.

The use of magnetic resonance (MR) imaging for the staging of lung cancer has also been studied [19, 20]. Although MR imaging has been shown to be valuable in assessing tumour extent in some patients, its diagnostic accuracy in mediastinal staging has not been shown to be better than that of $\mathrm{CT}$, with a mean sensitivity of $60 \%$. Using morphological imaging (CT and MR), the probability of mediastinal metastases increases with lymph node size. However, over one-third of lymph nodes that are $2-4 \mathrm{~cm}$ in small-axis diameter are hyperplastic and do not contain metastases [21, 22]. For these reasons, mediastinoscopy is often required to better clarify the clinical staging of the disease in the mediastinum.

In contrast to CT and MR, which depend on morphological criteria, PET allows a metabolic evaluation of the tissue. In particular, several in vitro and in vivo studies of lung cancer with PET-FDG have consistently demonstrated significantly increased uptake in all lung cancer cell types [10, 23, 24].

The main objective of this preliminary study was to determine whether PET-FDG allowed a more accurate classification of mediastinal staging than CT. The results suggest that PET-FDG is more accurate $(\mathrm{p}<0.05)$ than CT in the preoperative assessment of mediastinal involvement in NSCLC. In particular, PET was more sensitive $(90 \%)$, with a higher positive predictive value (90\%), than CT for mediastinal staging. PET-FDG has shown its ability to demonstrate tumour metabolism in normal-sized lymph nodes. In the present study, PETFDG correctly identified nodal metastases in seven patients with otherwise normal lymph node size but with histological proof of nodal involvement. However, four cases showed a lesser degree of mediastinal involvement despite histologically proven invasion of lymph nodes. Indeed the detectability of tumour involvement with PET scan depends not only on the size of a lesion but also on the concentration of radiotracer. It is likely that a critical mass of metabolically active malignant cells is required for PET diagnosis. Thus, if a node has normal size, it must have an intense FDG uptake for its detection by PET [24].

In the present series, there were four patients with a higher degree of mediastinal invasion as assessed by PET compared to histology; in each case, the tumour was centrally situated and led to a variable degree of bronchial obstruction. In our experience, analysis of PET data appears to be less conclusive in the case of a central tumour. Firstly, it may be difficult to separate the primary tumour from an adjacent nodal metastasis, and, secondly, bronchial obstruction can lead to a subacute inflammation in locoregional lymph nodes, with subsequent 18 -FDG uptake.

The findings of $86 \%$ specificity and $90 \%$ sensitivity for PET-FDG in determining $\mathrm{N}$ stage are comparable to those obtained in two recent studies with a smaller patient population [25, 26]. WAHL and co-workers [25] concluded that PET was more accurate $(81 \%)$ than CT $(52 \%)$ in staging disease in the mediastinum in patients $(n=33)$ with NSCLC. In their paper, the authors discussed different technical aspects of PET study and suggested that quantification and/or a combination of the anatomical and metabolic imaging data could increase accuracy in the future. In our opinion, semiquantitative analysis needs further evaluation for this indication, because there is already significant variability in normal mediastinal FDG uptake, which is dependent on different uncontrolled parameters (variability of local FDG distribution, degree of inflammation in bronchial airways, etc). In contrast, we agree that adding anatomical CT findings to metabolic PET findings could help localize metabolic activity in some patients and increase the accuracy of the method, particularly in the case of a central tumour.

More recently, CHIN et al. [26] focused their attention on N2 disease, and concluded that mediastinal uptake of FDG correlated with the extent of mediastinal involvement in NSCLC $(n=30)$. However, they suggested that PET-FDG results would not replace the need for histological confirmation because there were falsepositive cases in PET studies. This is probably true, at least until more experience is gained and technical improvements are made to the new technique.

In conclusion, we believe it may be possible to significantly improve the accuracy of nonsurgical mediastinal staging of non-small cell lung cancer by a combination of computed tomography and positron-emission tomographic imaging. Indeed, on the basis of our preliminary 
results, positron emission tomography with 18-fluorodeoxyglucose appears to be more sensitive than computed tomography in this indication; it justities multicentre trials to establish the reproducibility of these results.

\section{References}

1. Minna JD, Higgins GA, Glatstein EJ. Cancer of the lung. In: Cancer Principles and Practice of Oncology. Philadelphia, PA, Lippincott, 1985; pp. 507-597.

2. Shields TW. The significance of ipsilateral mediastinal lymph node metastasis (N2 disease) in non-small cell carcinoma of the lung. Thorac Cardiovasc Surg 1990; 99: 48-53.

3. Mc Loud T, Bourgouin P, Greenberg R, et al. Bronchogenic carcinoma: analysis of staging in the mediastinum with CT by correlative lymph node mapping and sampling. Radiology 1992; 182: 319-323.

4. Musset D, Grenier P, Carette MF, et al. Primary lung cancer staging: prospective comparative study of MR imaging with CT. Radiology 1986; 160: 607-611.

5. Georgian D, Rice T, Metha A, Wiedemann H, Stoller J, O'Donovan P. Intrathoracic lymph node evaluation by CT and MRI with histopathologic correlation in nonsmall cell bronchogenic carcinoma. Clin Imaging 1990; 14: $35-40$.

6. Webb R, Gatsonis C, Zerhouni E, et al. CT and MR imaging in staging non-small cell bronchogenic carcinoma: report of the radiologic diagnostic oncology group. Radiology 1991; 178: 705-713.

7. Hawkins R, Hoh C, Dahlbom M, et al. PET cancer evaluations with FDG. J Nucl Med 1991; 32: 1555-1558.

8. Rego S, Hoh C, Glaspy J, Aberle D, et al. Imaging of pulmonary mass lesions with whole-body positron emission tomography and fluorodeoxyglucose. Cancer 1993; 72: 82-90.

9. Warburg O. On the origin of cancer cells. Science 1956; 123: 309-314.

10. Nolop K, Rhodes C, Brudin H, et al. Glucose utilization in vivo by human pulmonary neoplasms. Cancer 1987; 60: 2682-2689.

11. Fujiwara T, Matsuzawa T, Kubota K, et al. Relationship between histologic type of primary lung cancer and carbon-11-L-methionine uptake with PET. J Nucl Med 1989; 30: 33-37.

12. Gupta N, Frank A, Dewan N, et al. Solitary pulmonary nodules: detection of malignancy with PET with 2-[F18]-fluoro-2-deoxy-D-glucose. Radiology 1992; 184: 441-444.

13. Dewan N, Gupta N, Redepenning L, Phallen J, Frick
M. Diagnostic efficacy of PET-FDG imaging in solitary pulmonary nodules. Chest 1993; 104: 997-1002.

14. Bury T, Paulus P, Corhay JL, et al. Apport diagnostique de la tomographie à émission de positons dans l'évaluation d'une opacité pulmonaire unique: étude préliminaire chez 30 patients. Med Nucl 1996; 20: 77-82.

15. Tisi G, Friedman P, Peters R, et al. Clinical staging of primary lung cancer. Am Rev Respir Dis 1983; 127: 659-664.

16. Cohen J. A coefficient of agreement for nominal scales. Educ Psychol Meas 1960; 20: 37-46.

17. Baron R, Levitt R, Sagel S, White M, Roper C, Marbarger J. Computed tomography in the preoperative evaluation of bronchogenic carcinoma. Radiology 1982; 145: 727732.

18. Dillemans B, Deneffe G, Verschakelen J, Decramer M. Value of computed tomography and mediastinoscopy in preoperative evaluation of mediastinal nodes in nonsmall cell lung cancer. Eur J Cardio Thorac Surg 1994; 8: $37-42$.

19. Patterson G, Ginsberg R, Poon P, et al. A prospective evaluation of magnetic resonance imaging, computed tomography and mediastinoscopy in the preoperative assessment of mediastinal node status in bronchogenic carcinoma. J Thorac Cardiovasc Surg 1987; 94: 679-684.

20. Grenier Ph, Dubray B, Carette M, Frija G, Musset D, Chastang C. Preoperative thoracic staging of lung cancer: CT and MR evaluation. Diagn Interv Radiol 1989; 1: $23-28$.

21. Libshitz H, McKenna R. Mediastinal lymph node size in lung cancer. Am J Roentgenol 1984; 143: 715-718.

22. McKenna R, Libshitz H, Moutain C, McMurtrey M. Roentgenographic evaluation of mediastinal nodes for preoperative assessment in lung cancer. Chest 1985; 88: 206-210.

23. Kubota K, Matsuzawa T, Fujiwara T, et al. Differential diagnosis of lung tumor with positron emission tomography: a prospective study. J Nucl Med 1990; 31 : 1927-1933.

24. Wahl R, Hutchins G, Buchsbaum D, Liebert M, Grossman B, Fisher S. 18-F-2-deoxy-2-fluoro-D-glucose uptake into human tumor xenografts: feasibility studies for cancer imaging with positron emission tomography. Cancer 1991; 67: 1544-1550.

25. Wahl R, Quint L, Greenough R, Meyer C, White R, Orringer M. Staging of mediastinal non-small cell lung cancer with FDG-PET, CT and fusion images: prospective preliminary evaluation. Radiology 1994; 191: 371-377.

26. Chin R, Ward R, Keyes J, et al. Mediastinal staging of non-small cell lung cancer with positron emission tomography. Am J Respir Crit Care Med 1995; 152: 20902096. 